\title{
Design of Intelligent Embedded System using Raspberry PI and Arduino for Industrial Maintenance
}

\author{
G.Sudhakar Reddy, V.Soma Laxmi
}

\begin{abstract}
Normally in industries there may be conventional network conversation s like RS232, RS485, CAN \& ZIGBEE and so on. Are confined for brief distances. This paper have grow to be aimed to analyze and set up an smart machine that have grow to be hired for Embedding, smart cellular telephone and internet based totally totally completely devices for business monitoring and controlling making use of Raspberry Pi and Arduino Uno. An ordeal and relative examination of eager tool turn out to be linked in a piece employer in this project. At some point of this undertaking we have been given that the device should show display enveloping surroundings situations collectively with sogginess, temperature, environment first-rate, and except actuate the manage devices like fumes fans, engine, switch and so on within the corporations. The essential detail of the framework is the potential to remotely deliver an alarm message, whilst a flame or immoderate temperature is perceived. Proper on the identical time because the closeness of smoke is excellent, the system will display an picture of the room popularity in a website web web page. This set up device can be required the client insistence to document the occasion to the Fireman the use of a manage sign. The upside of the use of this machine is that it'll decrease the possibility of false aware distinct of the Fireman. The system modified into furthermore visible to be appealing for any systems and farmers likewise to apply, as they'll feasibly manipulate the estate everywhere at something issue, achieving rate decline, asset saving, and profitable control in enterprise management. The software program code might be advanced in private domestic web page thru python programming language, for showing the output WebPages are superior with the resource of HTML.
\end{abstract}

\section{INTRODUCTION}

Modbus is simple, robust and only a serial communication protocol used along with its programmable logic controllers. This Modbus is not defined for physical layer, does not provide security for data and does not have standard format. So we require a simple yet robust network is needed which overcomes these problems i.e CAN. Controlled Area Network (CAN) is used in factory automation, medical, marine, military and anywhere. Originally, Controlled Area Networks (CAN) were developed for the automotive market to reduce the weight and cost of wiring harness and now it is also been used in Industries to add additional capabilities. But still we $\mathrm{r}$ facing problem, where we can't access the processed industrial data on web servers .Hence we are using the Arduino and Raspberry Pi combination, to rectify and provide web server based automation in Industries. Industrial automation issueof system and information development for control of

\footnotetext{
Revised Manuscript Received on September 10, 2019.

Mr.G.Sudhakar Reddy, Assistant Professor, Department of Electronics and Communication Engineering, CMR InstituteofTechnology, Hyderabad, Telangana,India. (Email : gsudhakar435@gmail.com)

(Email: gsudhakar435@gmail.com)

V.Soma Laxmi, Assistant Professor, CMR Institute of Technology, Hyderabad, Telangana, India. (Email: vasasomalaxmi431ece@gmail.com)
}

Industrial machines. Industrial robotization achieves comfort, essentialness adequacy, and security advantages prompting improved personal satisfaction. The distinction of framework engaged Industry robotization has been expanding enormously as of late because of straightforwardness and a lot higher moderateness. Also, with the quick extension of the Web, there is the potential for the remote control and observing of such system empowered apparatuses. But still we are facing problem, where we can't access the processed industrial data on web server.Hence we are using the Arduino and Raspberry Pi combination, to rectify and provide webserver based automation in Industries.

\section{METHODOLOGY}

The architecture of Raspberry $\mathrm{Pi}$ and Arduino is understood including the ports and GPIO pins. The pin descriptions and architectures of ARM11 and ATmega328 are understood. The raspberry pi board is instated utilizing the IP address of the board. Raspberry pi is activated using login id and password. The python code for Industry automation is written and executed in Raspberry pi. Raspberry Pi is interfaced with the relays which represent Industry appliances.

The Arduino IDE is learnt. The Arduino is initialized by setting serial port of the device. The code is written in Arduino IDE and uploaded in Arduino Uno board. Bluetooth module and relays are interfaced to Arduino board. Both the systems are tested for satisfactory operations.

\section{Associated paintings}

This studies has targeted at the utilization of present day technology to help manipulate animal cultivating, because of this ranch control automation in specific processes. Manakant Intarakamhaeng and etal considered the model of ranch management automation technology with RFID, end result; the appropriation of RFID, or radio-recurrence distinguishing evidence of gadgets and animals including 5 sorts of animal: cows, wild ox, sheep, pigs and bunnies were successfully absolutely diagnosed and recorded mechanically.

MdSaifudaullah field Bahrudin and Rosni Abu Kassim displayed an alarm tool in a non-prevent monitoring device that diagnosed the nearness of smoke considerable all round due to flame and stuck photographs through a virtual digital 
digicam delivered internal a room even as a flame takes place. The implanted structures used to build up this alarm machine were Raspberry $\mathrm{Pi}$ and Arduino Uno. The key element of the system is the capability to remotely deliver a geared up while a flame is identified. At the aspect at the equal time because the nearness of smoke is recognized, the device will display a image of the room united states of america in an internet net web page. The device may require the purchaser affirmation to document the occasion to the Fireman utilizing a quick Message management (SMS). The upside of using this system is that it'll decrease the possibility of fake conscious delivered of the Fireman. The camera will just capture a picture, so this tool will burn up best a little storage and strength.

There are numerous new era that can be applied in an increasingly more gifted management of ranches. The creators test has centered at the significance of present day era of Raspberry $\mathrm{Pi}$, Arduino and advanced mobile cellphone joined with bird cultivating.

\section{LAYOUT OF BLOCK DIAGRAM AND DESCRIPTION:}

The discern 3.1 and 3.2 bellow indicates the schematic \& rectangular outline of the monetary Automation device the use of Raspberry pi \& Arduino Micro controller with some inputs and output assets.

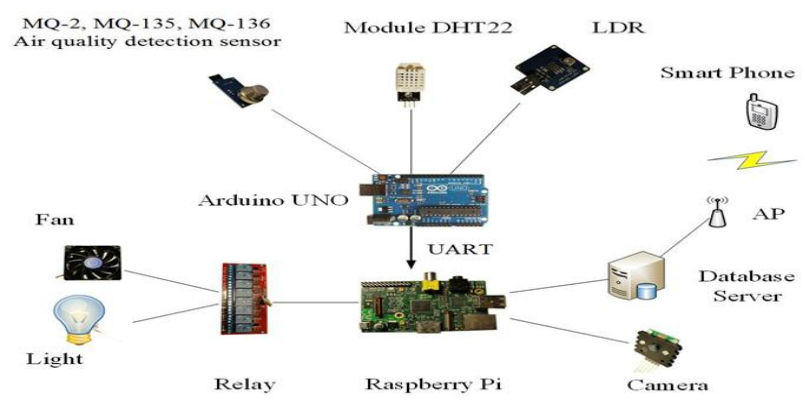

Fig: 3.1 square outline of Industrial Monitoring System

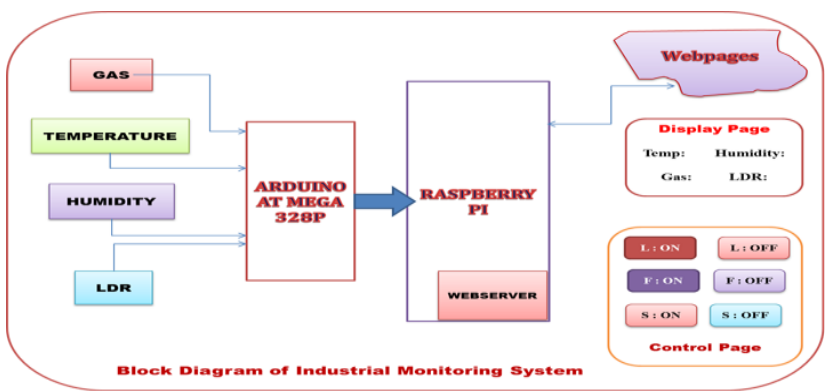

Fig: 3.2 Block Diagram of Industrial Monitoring System

The below figure describes the transmitter section and Control (Receiver) section of the industrial Automation system consisting of Input section of different sensors connected to Arduino ATMEGA328 microcontroller .This microcontroller is intern connected to the other microcontroller Raspberry $\mathrm{Pi}$ in which received data from Arduino in order. This data is stored in Raspberry Pi web server which can be accessed by any WebPages served system.

\section{A.Raspberry Pi}

Raspberry Pi [7] is a piece laptop board chipping away at the Linux running machine which connects to a pc show screen, console, and mouse. Raspberry Pi can be related to an virtual form and programming device artwork, it can likewise crammed in as a computer and Apache Webserver, MySQL can be delivered inside the board.

A GPIO [10] pin can be carried out as each a computerized statistics or a complex yield, and each art work at $3.3 \mathrm{~V}$. In evaluation to the Arduino, the Raspberry Pi which does now not have any clean assets of records. For that you need to employ an outer smooth to-superior converter (ADC) or be a part of the Pi to an interface board must be applied.

\section{B. Arduino}

Arduino is an open-supply microcontroller precise with created degrees. The controller indicates up not to be expensive and uses low electric power, five.Five volts. C and $\mathrm{C}++$ were carried out for this development. Arduino can connect to a pc via the All-inclusive Sequential transport (USB) and perform with exceptional related frill in each easy sign and superior sign.

The Arduino [9] is a microcontroller degree, mounted on a board that fittings efficiently into typically computers. It allows the consumer to software program the regionally to be had Atmega chip to do various things with programming language, in obligations known as attracts.

\section{Humidity Sensor module}

Natural situations straightforwardly impact animal commercial corporation including to 3 ceaseless epidemics, as an example, winged creature Influenza and Hand Foot and Mouth contamination. In this manner, DHT22 is find out as a blue pencil for estimating temperature (for each Fahrenheit and Celsius properly worth) and humidity. The estimation unit may be exhibited in a computerized signal form.

\section{Fuel Sensor module}

The module fills in as an Air quality identification gasoline Sensor, this is sensitive to gas unsafe to human, connected to quantify NH3, NOx, Alchohol, Benzene, CO, and $\mathrm{CO} 2$. The module is also applied for controlling climate conditions and air cleaners in systems. The estimation unit is displayed in a clean sign.

On this exploration, three manage structures were applied, comprising of MQ-2, MQ-a hundred thirty five, and MQ-136 because of its gas estimation contrasts.

\section{E. Photosensitive sensor module (LDR)}

A mild sensor turn out to be carried out for estimation of mild stress particularly for unaided eye mild, its unit is referred to as Lux [8].Mild Subordinate Resistor (LDR) is a moderate sensitive opposition converting digital obstruction on the same time as there may be a moderate frequency, known as photo Resistor or image Conductor. The resistor became produced the usage of Semiconductor, Cadmium Sulfide (Cds) or Cadmium Selenide (CdSe). Those

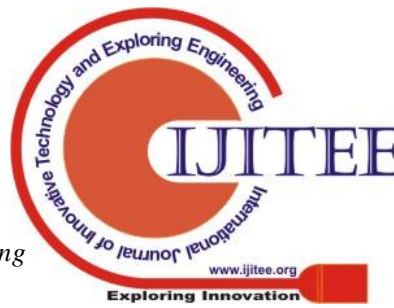


materials are semiconductors included in an earthenware sheet as a base.

\section{F. Hardware join}

Raspberry Pi and Arduino were connected thru UART. The relationship changed into a sequential communication as complete Duplex thinking about there has been -tactics that records might be transmitted with the resource of pin TX and RX.

An immediate connection many of the Raspberry Pi and Arduino modified into disallowed, because of its electric functionality contrasts, this is three. 3 volts for the Raspberry $\mathrm{Pi}$ and five volts for the Arduino. Bi-directional reason stage Converter have to be applied to isolate them.

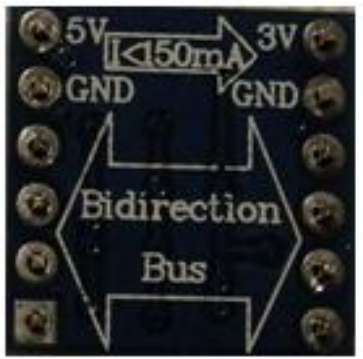

Fig.2: Bi-directional common sense degree Converter.

A connection among a virtual digicam and Raspberry $\mathrm{Pi}$ with the useful aid of applying regular device Interface (CSI) fills in as a thing-to-thing connection, giving a quick records transmission and low energy usage.

MJPG-Streamer is an important utility command duplicating data from a solitary contribution to numerous yields. A image may be exhibited in a device getting to from a web program on a laptop. In this examination, a photograph from a virtual camera might be taken to expose on a complex cellular. All sensors will be associated with the aid of way of Board Arduino and the information might be transmitted from UART to Board Raspberry Pi. Raspberry Pi fills in as a controller of a ventilator, telling a running scenario to the advanced cell and filled in as an statistics sender to preserve in a server computer.

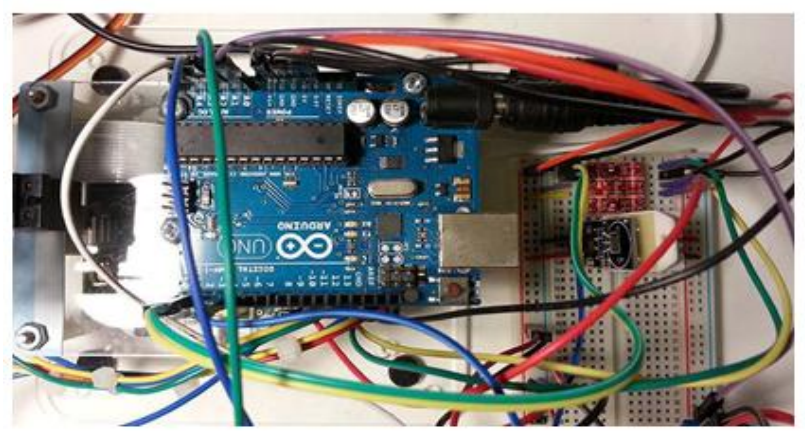

Fig. Three: suggests the relationship of the circuit.

\section{G. Software application}

A connection among Raspberry Pi and advanced mobile telephones became researched in this research. Programming of a conversation among a server and a purchaser made out of factors of view. The Raspberry Pi might check whether or not or now not there have been any related customers, furnished that this is proper, a shared information transmission can be completed. By way of doing this, an application might ship the records by means of the usage of Attachment alluding to IP deal with and Port in vehicle Layer the usage of TCP protocol.

Linux modified into the essential working device implemented for Raspberry IP. In this exploration, Raspbian Wheezy, an powerful jogging device affirmed with the useful resource of the maker, have become added on a SD Card through software program Win32 Circle manager After the status quo, IP address format, digital digital camera setup, UART connection, and Apache Webserver set up order is probably directed. Fig.Four: Flowchart of the Python programming in Raspberry Pi.

Python writing laptop applications is installation for the development of Raspberry Pi. Python may want to peruse the Arduino signal truly honestly really worth by manner of UART and then accumulate the obtained sign to the database for dealing with. At the off danger that the really actually really worth outperformed component, the over sign would be sent to GPIO pins to conscious the clean signal. If there need to be an prevalence of remarkable records, a "high" signal may be sent to GPIO pin 17 and the ventilator can also delete the internal air (Fan out on). If there ought to be an incidence of excessive temperature, a "excessive" sign will be despatched to GPIO pin 27 and the ventilator could art work robotically (Fan in on). In case of glow alternate, the data might be despatched to GPIO pin 22 and electric powered powered lighting fixtures is probably opened. Advantageously, running of adornments can be customized by using the usage of the client as referenced in Fig. 4 speaking the flowchart of the Python programming in Raspberry Pi.

Some other vast thing is this superior mobile telephone works with the Android walking tool. Created packages are at the Android running machine using the Java language and interfaced with the Raspberry Pi through the a ways flung tool. This could take the motivation from the Arduino to peruse suggests, as an example, temperature, humidity, mild, toxic gases, and so forth. It is organized to govern fans and lighting fixtures, and can be observed thru the net at any time.

\section{EXPERIMENTAL results AND COMPARISONS}

Fig four.1 indicates the experimental setup of Raspberry $\mathrm{Pi}$ and Arduino based definitely truly business Automation system. In this venture we are the use of Arduino microcontroller it truly is associated with one-of-a-type sensors which gather the sensor records, technique the information and transmit the processed information to Arduino microcontroller which intern switch the processed information to Raspberry pi through serial cable. The three transfers are associated with the Arduino board which are interfaced to Raspberry Pi board. Arduino is applied as a transitional for offering $5 \mathrm{v}$ of supply to transfers as yield of Raspberry is most effective $3.3 \mathrm{v}$. 


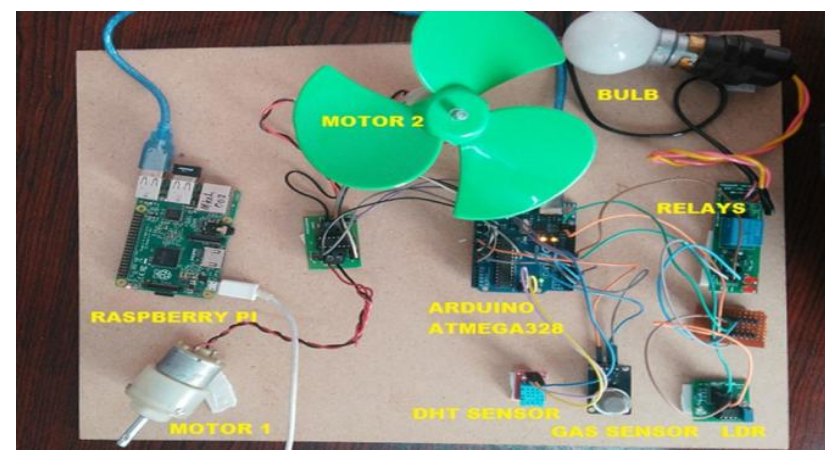

Fig : 4.1 Working experimental setup for Industrial Automation System

When one of the parameter in industry is increased abruptly then an indication goes to Raspberry pi and concerned control unit(motor) gets activated automatically .Incase if we want to control manually, we can also control by pressing the concerned button present on control unit page with subject ON1 is sent to Raspberry pi account, light 1 is switched $\mathrm{ON}$ and a response for the same is shown in next immediate tab. Similarly ON2, ON3, OFF1, OFF2, ALL ON, ALL OFF are shown in the new tabs for Industry Automation Fig 5.2 and Fig 5.3 shows the response given while performing the action on particular device Status. Eg.Turned ON/OFF.
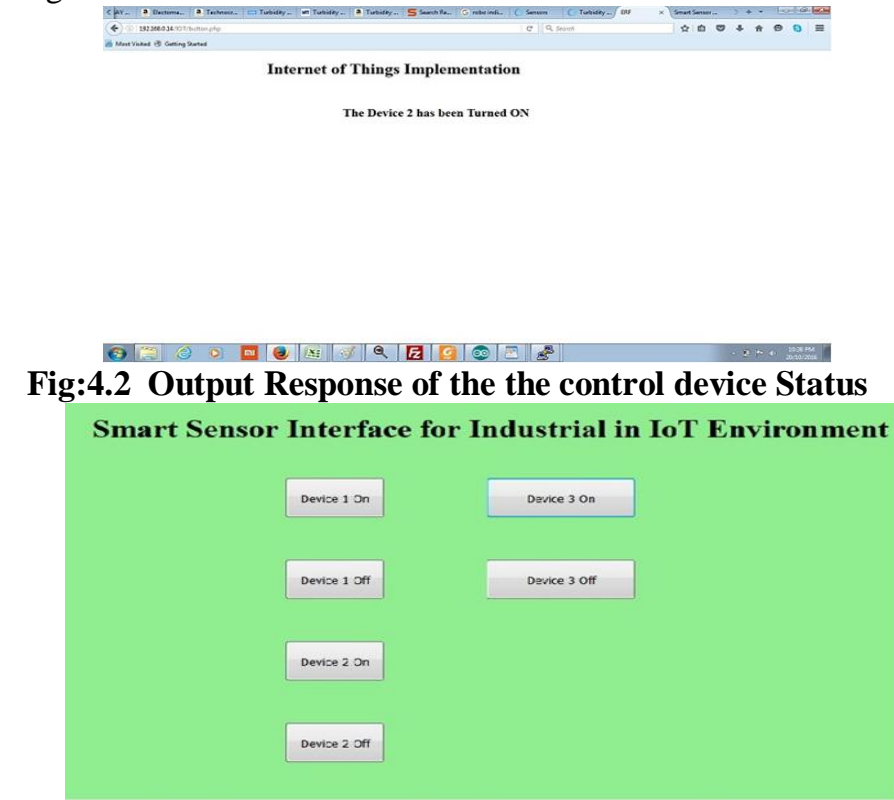

Fig:4.3 Webpage to control the devices

The figure bellow describes the output status of all the sensor inputs connected to Arduino and Raspberry Pi.

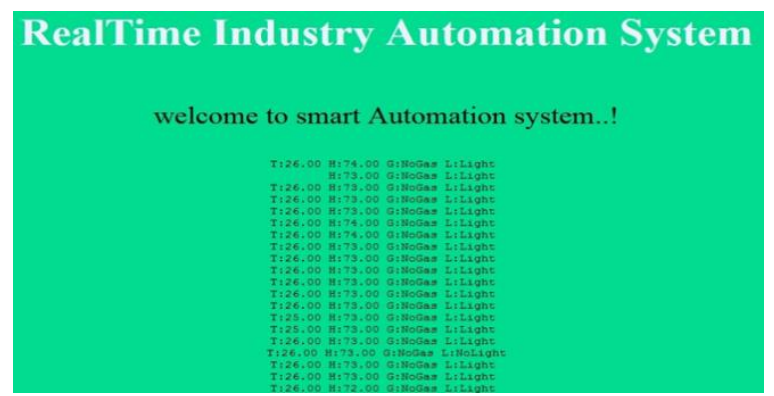

Fig: 4.4 Output Status of Sensors on webpage
This figure describes display outputs of the sensors serially, which are programmed with python language on Raspberry Pi

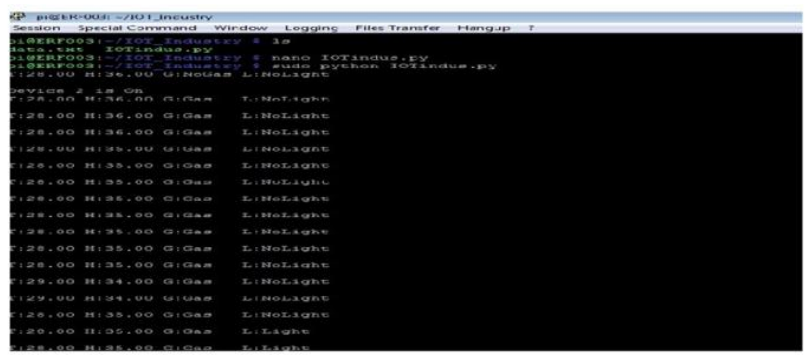

Fig:4.5 Output status of Sensor at Raspberry pi end

\section{CONCLUSION AND FUTURE SCOPE}

This inserted system is inventive for Industrial applications which change the customary ranch to a Savvy Homestead. Furthermore, the system Need to chip away at makes use of of net server primarily based definitely in reality software assisting the monetary uses to control and screen constant situation settings, as an instance, temperature, weather scenario and first-class ,humidity, light power and channel fan switches. This astute device can lessen the price, time and paintings and could be very benevolent for Industrialists. In coming destiny Raspberry Pi model B should be modified into Raspberry Pi 2 due to its extra viability and server working decrease. All gathered commercial packages statistics ought to be despatched from server and stored in some other device. Extra over a stay stock feeding gadget want to be additionally superior to make this greater whole tool.

\section{REFERENCES}

1. www.Instructables.Com/...Net web site...Raspberry-pi

2. https://www.Raspberrypi.Org/

3. www.Element14.Com/network/network/raspberry-pi

4. elinux.Org/RPi_Hub

5. www.Treehugger.Com/.../20-top notch-responsibilitiesraspberry-pi

6. "Raspberry Pi Compute Module: new product!". Raspberrypi.Org.

7. Kelion, Leo (2 February 2015). "Raspberry Pi 2 unveiled with quicker processor and extra memory". BBC facts. Retrieved 3 February 2015.

8. "everyday overall performance - measures of the Raspberry Pi's common normal performance.". RPi standard standard performance. ELinux.Org. Retrieved 30 March 2014.

9. Benchoff, Brian. "sixty four RasberryPis have grow to be a supercomputer". Hackaday. Retrieved 30 March 2014.

10. "Raspberry Pi, supported video resolutions". ELinux.Org. 30 November 2012. Retrieved eleven December 2012.

11. "Introducing Raspberry Pi model B+". Raspberry Pi basis Retrieved 14 july 2014

12. "Raspberry Pi Compute Module: New Product!". Raspberry Pi foundation. Retrieved 22 September 2014.

13. "New video features! MPEG-2 and VC-1 decode, H.264 encode, CEC". Raspberry Pi basis. Retrieved 26 August 2012

14. "SMSC LAN9512 net website online;". Smsc.Com. Retrieved 6 may additionally 2012

15. "Microchip/SMSC LAN9514 records sheet;"(PDF). Microchip Retrieved 15 July 2014 